(C) The Authors 2019. This is an Open Access article, distributed under the terms of the Creative Commons Attribution licence (http://creativecommons.org/licenses/by/4.0/), which permits unrestricted re-use, distribution, and reproduction in any medium, provided the original work is properly cited.

\title{
Oligosaccharides in goats' milk-based infant formula and their prebiotic and anti-infection properties
}

\author{
Andrea Leong ${ }^{1}$, Zhiqian Liu $^{2}$, Hala Almshawit ${ }^{1}$, Bogdan Zisu ${ }^{1}$, Christopher Pillidge ${ }^{1}$, Simone Rochfort ${ }^{2}$ and \\ Harsharn Gill ${ }^{1 *}$ \\ ${ }^{1}$ Biosciences E Food Technology Discipline, School of Science, RMIT University, Plenty Road, Bundoora, VIC 3083, Australia \\ ${ }^{2}$ Biosciences Research Division, Department of Environment and Primary Industries, AgriBiosciences, 5 Ring Road, Bundoora, \\ VIC 3083, Australia \\ (Submitted 2 January 2019 - Final revision received 24 April 2019 - Accepted 19 May 2019)
}

\section{Abstract}

Human milk contains an abundant supply and diverse array of oligosaccharides that are known to impart significant health benefits to the nursing infant including establishment and maintenance of a healthy gut microflora, immune development and protection against gastrointestinal infections. When breastfeeding is not possible or insufficient, infant formulas are commonly used as an alternative. However, limited information is available about the presence of naturally occurring oligosaccharides in these infant formulas and their likely health benefits. The present study examined the presence of naturally occurring oligosaccharides in commercial goats' milk-based stage 1 and stage 2 infant formulas and their prebiotic and anti-infection properties. LC/MS was used to detect and quantify oligosaccharides and their prebiotic potential was assessed by their ability, at concentrations present in reconstituted ready-to-use infant formula, to promote the growth of Bifidobacterium animalis subsp. lactis BB12, B. longum BB536, Lactobacillus acidophilus 4461 and L. casei 2607 in vitro. For anti-infection properties, the ability of goat milk oligosaccharides to prevent the adhesion of Escherichia coli NCTC 10418 and a Salmonella typhimurium isolate to Caco-2 cells was investigated. The results showed the presence of fourteen quantifiable oligosaccharides in stage 1 and stage 2 goats' milk-based infant formula. This was similar to the number of oligosaccharides detected in the fresh goats' milk. Of these, five were structurally similar to those found in human milk. These oligosaccharides were shown to significantly enhance the growth of bifidobacteria and lactobacilli and reduce the adhesion of E. coli NCTC 10418 and S. typhimurium to Caco-2 cells. Together, these results suggest that oligosaccharides naturally present in goats' milk-based infant formula exhibit strong prebiotic and anti-pathogen adhesion properties and may confer gut health benefits to infants.

Key words: Oligosaccharides: Goats' milk infant formula: Liquid chromatography/mass spectrometry: Prebiotic effects

Breast milk is considered a complete food for the newborn and breastfeeding is known to provide a wide range of health benefits to both mother and the baby. The WHO recommends exclusive breastfeeding for the first 6 months of an infant's life, and continued breastfeeding with complementary foods for up to 2 years of age ${ }^{(1)}$. However, when a mother's milk is not available, infant milk formula is considered as an effective alternative. Cows' milk-based infant formulas are widely used, but goats' milk-based products are becoming increasingly popular as goats' milk is considered to be more similar to human milk compared with cows' milk. This includes higher levels of oligosaccharides in goats' milk relative to milk of other mammalian species $^{(2)}$ and lower levels of $\alpha$-S1 casein ${ }^{(3)}$. Goats' milk is also reported to exhibit significant homology in lactoferrin $\mathrm{N}$-glycans with human milk ${ }^{(4)}$.

Human milk is known to contain high concentrations (5-20 g/l in mature milk) and diversity of oligosaccharides ${ }^{(5,6)}$. Several studies have shown that oligosaccharides, particularly human milk oligosaccharides (HMO), are effective in selectively promoting the growth of bifidobacteria and shaping the composition of intestinal microbiota, preventing adhesion of pathogens to intestinal mucosa and reducing the risk of bacterial, viral and parasitic infections, modulating immune and intestinal immune cell function, lowering the risk of necrotising enterocolitis and providing nutrients (sialic acid) for brain development and cognition $^{(7-12)}$. Especially, 2'-fucosyllactose (2'-FL) has been

Abbreviations: 2-FL, 2-fucosyllactose; GOS, galacto-oligosaccharides; HMO, human milk oligosaccharides; NCTC, National Collection of Type Cultures; OD $_{600 \mathrm{~nm}}$, optical density at $600 \mathrm{~nm}$; S1-GIF, stage 1 goat milk infant formula; S2-GIF, stage 2 goat milk infant formula. 
reported to be important in protecting infants against gut infections ${ }^{(13,14)}$. This has stimulated efforts by infant formula manufacturers to produce infant formulas that closely resemble human milk in composition and performance ${ }^{(15)}$. However, this process is challenging given that oligosaccharides in goats' or cows' milk exhibit limited diversity and are present at significantly lower concentrations compared with those in human milk ${ }^{(16-20)}$. Importantly, the complexity of HMO makes it almost impossible for these to be duplicated in infant formulas ${ }^{(21)}$. To overcome this challenge, modern infant formulas are commonly fortified with fructo-oligosaccharides and galactooligosaccharides (GOS) ${ }^{(22)}$. However, their effectiveness in conferring health benefits similar to those of breast milk remains to be proven ${ }^{(23,24)}$

It has previously been shown that high heat treatment could cause degradation or change to the structure of some oligosaccharides $^{(25,26)}$. How the heat treatments applied during manufacture of infant formula (milk pasteurisation and exposure to temperatures of $180-220^{\circ} \mathrm{C}$ during spray drying ${ }^{(27)}$ ) affect the presence and functionality of naturally present or added oligosaccharides in infant formula remains unknown. Only a handful of studies to date have attempted to quantify the amount and types of milk oligosaccharides present in infant formula products. The primary objective of the present study was to investigate the diversity and concentration of oligosaccharides present in goats' milk and goats' milk-based infant formula and their prebiotic and anti-pathogen adhesion properties.

\section{Materials and methods \\ Oligosaccharide standards}

Oligosaccharide standards were purchased from Dextra Laboratories. These included: 3'-sialyllactose, 6'-sialyllactose, 3'-sialyl- $N$-acetyllactosamine, 6'-sialyl- $N$-acetyllactosamine, 2'-FL, 3'-fucosyllactose, lacto- $N$-hexaose, 3'-galactosyllactose, 4'-galactosyllactose, 6'-galactosyllactose and disialyllactose. Oligosaccharide standards which were received in powder form were reconstituted with Milli-Q water $\left(22 \pm 2{ }^{\circ} \mathrm{C}\right)$ to make a stock solution at a concentration of $1 \mathrm{~g} / \mathrm{l}$. Stock solutions were further diluted with Milli-Q water to give a final concentration range of $0 \cdot 1-0 \cdot 001 \mathrm{~g} / \mathrm{l}$.

\section{Preparation of oligosaccharides}

Oli6 stage 1 (S1-GIF) and stage 2 goats' milk infant formulas (S2-GIF) and raw goats' milk (pooled milk from a group of ten Saanen goats) were obtained from Nuchev Pty Ltd. The GOS mixture was purchased from New Francisco Biotechnology Corporation Ltd (King-Prebiotics® GOS-700-P, batch number 17003). GIF was reconstituted according to manufacturer's instructions. GOS was reconstituted to $20 \mathrm{~g} / \mathrm{l}$, whilst concentrations of milk oligosaccharides extracted from infant formula were adjusted to match typical levels in reconstituted GIF. Milk samples were centrifuged at $4000 \boldsymbol{g}$ for $30 \mathrm{~min}$ at $4^{\circ} \mathrm{C}$ to remove remaining milk lipids. Equal volumes of MilliQ water were added to defatted milk and subsequently filtered through a $10 \mathrm{kDa}$ molecular weight cut-off filter (Amicon® Centrifugal Filters) in a centrifuge ( $4000 \boldsymbol{g}$ for $30 \mathrm{~min}$ ). The clear filtrate was assumed to contain all oligosaccharides. Extracts were filtered with Millipore $0.45 \mu \mathrm{m}$ syringe filter.

\section{Oligosaccharide quantification by LC/MS}

Analysis of oligosaccharides was performed based on the protocols of Liu et $a l^{(28)}$; this method was found to be highly accurate and sensitive in detecting oligosaccharides (limit of detection of $<0.1 \mathrm{ng}$ and accuracy of $95-105 \%$ for a variety of spiked oligosaccharides).

Chromatographic separation of milk oligosaccharides was achieved via a Kinetex hydrophilic Interaction liquid chromatography column $(150 \times 4.6 \mathrm{~mm}, 2.8 \mu \mathrm{m}$, Phenomenex $)$ on an Agilent 1290 Infinity HPLC system (Agilent). Components of the LC/MS included a degasser, binary pump, temperature controlled auto-sampler (maintained at $4^{\circ} \mathrm{C}$ ) and column compartment (maintained at $30^{\circ} \mathrm{C}$ ). The mobile phase $(\mathrm{A}+\mathrm{B})$ consisted of water containing $5 \mathrm{~mm}$ ammonium acetate (A) and acetonitrile with $0 \cdot 1 \%$ formic acid (B). The flow rate was $0.6 \mathrm{ml} / \mathrm{min}$ with a gradient elution of 3-50\% of solution (A) over $35 \mathrm{~min}$. Analyte detection was performed via mass spectrometry LTQ Orbitrap Velos (Thermo Scientific) with a heated electrospray ionisation source maintained at $270^{\circ} \mathrm{C}$ by a heating source of $350^{\circ} \mathrm{C}$. The sheath, auxiliary and sweep gases were at 40,15 and 8 units, respectively. Voltage source was fixed at $3.2 \mathrm{kV}$ in negative mode. Data were collected in profile data acquisition mode over the mass range from 200 to 2000 mass/charge $(\mathrm{m} / \mathrm{z}$ ) in negative Fourier transform mode (resolution of 60 000) and processed using the Xcalibur software package (Thermo Scientific).

\section{Evaluation of prebiotic properties}

Two Bifidobacterium strains (B. animalis subsp. lactis BB12 and B. longum BB536) and two Lactobacillus strains (L. casei 2607 and $L$. acidophilus 4461) were used in these experiments ${ }^{(29-31)}$. Strains were grown anaerobically in De Man, Rogosa and Sharpe (MRS) broth ${ }^{(32)}$ with filter-sterilised cysteine $(0.05 \%$, final concentration) added for the Bifidobacterium strains. For growth experiments, modified MRS was used in which glucose was omitted unless otherwise indicated. Lactose ( $5 \mathrm{~g} / \mathrm{l}$ ) was also included as a positive control. Bacterial suspensions of log-phase cells in $2 \times$ modified MRS were adjusted to an optical density at $600 \mathrm{~nm}$ $\left(\mathrm{OD}_{600 \mathrm{~nm}}\right)$ of $0 \cdot 11$, and then $100 \mu \mathrm{l}$ of the cell suspension was added to wells in a sterile ninety-six-well plate. Each well was pre-filled with an equal volume of milk oligosaccharide extract and gently mixed after addition of cells. The final oligosaccharide concentration was adjusted to match that of the reconstituted goats' milk-based infant formula (ready-to-use as per the manufacturer's instructions) in order to maintain the same concentration and relative proportions of the oligosaccharides. Plates were incubated anaerobically at $37^{\circ} \mathrm{C}$ for $24 \mathrm{~h}$. $\mathrm{OD}_{600 \mathrm{~nm}}$ readings of the suspensions were taken before and after growth using a plate reader. The extent of growth was determined by the increase in $\mathrm{OD}_{600 \mathrm{~nm}}$ from $t=0$. 
Each strain/substrate combination and controls in wells were assayed in triplicate. Sterile GOS (20 g/l) was included as the positive control, while negative controls contained only bacteria and the medium without added oligosaccharides. Dextrose and lactose were used as comparison benchmarks for simple sugar utilisation by the bacteria.

\section{Cell culture}

Caco-2 cells, human colorectal epithelial adenocarcinoma cells which exhibit high cellular differentiation, were selected as a human gut adhesion model. The Caco- 2 cells were cultured in Dulbecco's modified Eagle's medium supplemented with $10 \%$ fetal bovine serum and $1 \%$ of an antibiotic-antimycotic solution (10 000 units $/ \mathrm{ml}$ of penicillin, $10000 \mu \mathrm{g} / \mathrm{ml}$ of streptomycin and $25 \mu \mathrm{g} / \mathrm{ml}$ of Gibco® Amphotericin B; Life Technologies). Cells were incubated at $37^{\circ} \mathrm{C}, 95 \%$ humidity in an atmosphere containing $5 \%$ carbon dioxide. At $80 \%$ confluence, cells were passaged as follows: after aspiration of culture media, cells were washed with $5 \mathrm{ml}$ pre-warmed PBS at $\mathrm{pH} 7$ (Life Technologies), $5 \mathrm{ml}$ of trypsin-EDTA $(0 \cdot 25 \%$, Life Technologies) was added and then the cells incubated for 5-10 min. Cells were washed with fresh media $(10 \mathrm{ml})$ and centrifuged at $815 \boldsymbol{g}$ for $5 \mathrm{~min}$ to remove trypsin and EDTA. The cell pellet was reconstituted with $1 \mathrm{ml}$ fresh media. A cell count was performed and the cells recultured in a sterile flask pre-filled with fresh media (density of $10^{5}$ cells $/ \mathrm{ml}$ ). For anti-adhesion studies, twenty-four-well plates were seeded with $5 \times 10^{5}$ cells per well and incubated as before to obtain confluent monolayers. At $1 \mathrm{~d}$ prior to experimentation, the media was replaced with fresh media without any antibiotic-antimycotic.

\section{Anti-adhesion study with Caco-2 cell monolayer}

The protocol used was based on those of earlier studies with slight modifications ${ }^{(33,34)}$. Escherichia coli National Collection of Type Cultures (NCTC) 10418 and Salmonella typhimurium were obtained from RMIT University's culture collection. A single colony isolate of each strain was inoculated into $9 \mathrm{ml}$ nutrient broth (Amyl Media) in a sterile tube and incubated at $37^{\circ} \mathrm{C}$ for $2 \mathrm{~h}$ with shaking for cells to reach exponential growth phase. Each suspension was then adjusted to an $\mathrm{OD}_{600 \mathrm{~nm}}$ of 0.3 and oligosaccharides (prepared as described previously) added at equal volumes (final oligosaccharide concentration equivalent to that of the ready-to-use reconstituted goats' milk-based infant formula) and the cell suspensions incubated at $37^{\circ} \mathrm{C}$ for $120 \mathrm{~min}$. In addition, sterilised GOS (20 g/l) and Milli-Q water were used as positive and negative controls, respectively. Lactose, in non-growth-limiting concentrations $(5 \mathrm{~g} / \mathrm{l})$, was also included as one of the treatments to distinguish its effect from oligosaccharides. The bacteria-oligosaccharide suspension was added to each well in a sterile twenty-four-well tissue culture plate containing a Caco- 2 monolayer and incubated aerobically at $37^{\circ} \mathrm{C}$ for $90 \mathrm{~min}$. Post-incubation, the Caco- 2 cells were washed three times with PBS and then lysed with cold $0 \cdot 1 \%$ Triton-X to release adherent bacterial cells. Serial dilutions of lysates were prepared and plated onto nutrient agar plates which were incubated anaerobically for $24 \mathrm{~h}$ or until colonies were evident. Numbers of colonies were then counted. Each experimental treatment was conducted in triplicate. Anti-adhesion percentage was calculated as:

$$
\begin{aligned}
& \text { Anti-adhesion }(\%)= \\
& \frac{\text { bacteria count }(\text { control })-\text { sample bacteria count }(\text { sample })}{\text { bacteria count }(\text { control })} \times 100 .
\end{aligned}
$$

\section{Statistical analysis}

Results for LC/MS analyses, and growth and adhesion assays are expressed as means with their standard errors. For experiments involving bacterial cultures, the difference between each treatment group was tested using one-way ANOVA and Tukey's multiple comparison tests. Statistical significance was defined as a $P$ value of $<0 \cdot 05$. All statistical analyses were performed using GraphPad Prism (GraphPad Prism version 8 for Windows, GraphPad Software).

\section{Results}

\section{LC/MS quantification of milk oligosaccharides}

The high throughput analytical method used in the present study was effective in identifying oligosaccharides present in significant quantities without requiring sample concentration. A total of fourteen oligosaccharides were found to be present in S1-GIF and S2-GIF. This included seven major (predominant) and seven minor (present in small amounts) oligosaccharides (Table 1; Figs. 1-3). 3'-galactosyllactose and 6'-galactosyllactose were grouped together as triose sugars due to their complex chromatograph profiles (Fig. 4). Of the fourteen oligosaccharides, five (2'-FL, 3'-sialyllactose, 6'-sialyllactose, lacto-N-tetraose and lacto-N-hexaose) were structurally similar to those found in human breast milk (Table 1). There was no difference in the chromatographic profiles of S1-GIF and S2-GIF (data not shown).

The oligosaccharide profile of goats' milk was similar in diversity to that of S1-GIF and S2-GIF with a few minor differences. One of the minor milk oligosaccharides, $\mathrm{N}$-acetyl-glucosaminyl-lactose $(\mathrm{m} / \mathrm{z} 544 \cdot 18)$ was found to be absent in raw goats' milk (Table 1, Fig. 3), and S2-GIF had slightly higher abundance of 6'-glycolyl-neuraminyl-lactose $(\mathrm{m} / z$ 648.19). Furthermore, the chromatographic profile of GOS was dominated by 3'-galactosyllactose and 6'-galactosyllactose that were also detected in goats' milk (Fig. 4). It also implies that the total concentration of triose sugars identified in the present study included GOS. For both GIF prepared according to the manufacturer's instructions, oligosaccharide concentrations were similar to those found in normal unpasteurised goats' milk (Table 1).

\section{Goat milk oligosaccharides promote Bifidobacterium and Lactobacillus growth}

As shown in Fig. 5, the growth rate of B. animalis BB12 and B. longum BB536 was significantly enhanced, compared with controls, when strains were co-cultured in the presence of oligosaccharides derived from S1-GIF and S2-GIF. This growthpromoting effect was also significantly greater $(P<0.5)$, to that observed with GOS. Oligosaccharides purified from S1-GIF and 
Table 1. Concentration of major and minor oligosaccharides detected in goats' milk-based infant formula and their presence in human milk (Mean values with their standard errors; relative percentages)

\begin{tabular}{|c|c|c|c|c|c|c|c|c|c|c|c|}
\hline \multirow[b]{2}{*}{ Oligosaccharides } & \multirow[b]{2}{*}{ Neutral mass $(\mathrm{m} / \mathrm{z})$} & \multicolumn{3}{|c|}{ S1-GIF } & \multicolumn{3}{|c|}{ S2-GIF } & \multicolumn{3}{|c|}{ Goats' milk } & \multirow{2}{*}{$\begin{array}{l}\text { Reported in human } \\
\text { milk }^{(35,43)}\end{array}$} \\
\hline & & Mean & SEM & $\%$ & Mean & SEM & $\%$ & Mean & SEM & $\%$ & \\
\hline \multicolumn{12}{|c|}{ Major milk oligosaccharides $(\mu \mathrm{g} / \mathrm{ml}$ and relative percentage) } \\
\hline 3'-SL & $633 \cdot 212$ & 7.67 & 0.084 & $36 \cdot 8$ & 6.33 & 0.087 & $32 \cdot 7$ & 3.24 & 0.070 & $21 \cdot 8$ & Y \\
\hline 6'-SL & 633.212 & 4.45 & 0.026 & 21.4 & 4.90 & 0.014 & $25 \cdot 3$ & 5.80 & 0.525 & $39 \cdot 1$ & $Y$ \\
\hline 2'-FL & $488 \cdot 174$ & 0.88 & 0.039 & $4 \cdot 22$ & 1.38 & 0.042 & $7 \cdot 14$ & 1.12 & 0.095 & 7.54 & $Y$ \\
\hline 6'-SLN & 674.238 & 0.12 & 0.000 & 0.58 & 0.15 & 0.001 & 0.78 & 0.28 & 0.040 & 1.89 & \\
\hline DSL & 924.307 & 0.42 & 0.004 & 2.02 & 0.44 & 0.001 & 2.28 & 0.31 & 0.050 & 2.09 & \\
\hline LNH & $1072 \cdot 381$ & 0.06 & 0.004 & 0.29 & 0.10 & 0.001 & 0.52 & 0.09 & 0.015 & 0.61 & $Y$ \\
\hline Triose* & $504 \cdot 169$ & 7.24 & 0.272 & 34.7 & 6.03 & 0.087 & 31.2 & 4.01 & 0.755 & 27.0 & \\
\hline \multicolumn{12}{|c|}{ Minor milk oligosaccharides (peak area) } \\
\hline NAL & $544 \cdot 18$ & 1609364 & 6706 & & 1134549 & 4695 & & ND & ND & & \\
\hline NGL $†$ & $648 \cdot 19$ & 23527858 & 258449 & & 33904550 & 314932 & & 48735130 & 63144 & & \\
\hline LNT & 706.23 & 158050 & 593 & & 167765 & 4951 & & 172551 & 2933 & & $Y$ \\
\hline 3'-SHL & 794.25 & 1311192 & 2447 & & 1109955 & 6827 & & 656108 & 1495 & & \\
\hline 3'-GNL & 689.22 & 676944 & 6397 & & 954586 & 1401 & & 2538648 & 18329 & & \\
\hline SNGHL & 810.25 & 724860 & 6527 & & 1045144 & 8320 & & 1225516 & 9202 & & \\
\hline SNGL & 939.29 & 556212 & 1132 & & 817156 & 391 & & 1133007 & 1986 & & \\
\hline
\end{tabular}

S1-GIF, stage 1 goat milk infant formula; S2-GIF, stage 2 goat milk infant formula; 3'-SL, 3'-sialyllactose; Y, yes; 6'-SL, 6'-sialyllactose; 2'-FL, 2'-fucosyllactose; 6'-SLN, 6'-sialyl$\mathrm{N}$-acetyllactosamine; DSL, disialyllactose; LNH, lacto- $N$-hexaose; NAL, $N$-acetyl-glucosaminyl-lactose; ND, not detected; NGL, glycolyl-neuraminyl-lactose; LNT, lacto- $N$-tetraose; 3'-SHL, sialyl-galactosyl-lactose; 3'-GNL, glycolyl-neuraminyl-lactosamine; SNGHL, $N$-glycolyl-neuraminyl-hexosyl-lactose; SNGL, sialyl- $N$-glycolyl-neuraminyl-lactose.

* Total putative concentration reported for 3'-galactosyllactose and 6'-galactosyllactose.

† Relative abundance reported as a total sum of two isomers.

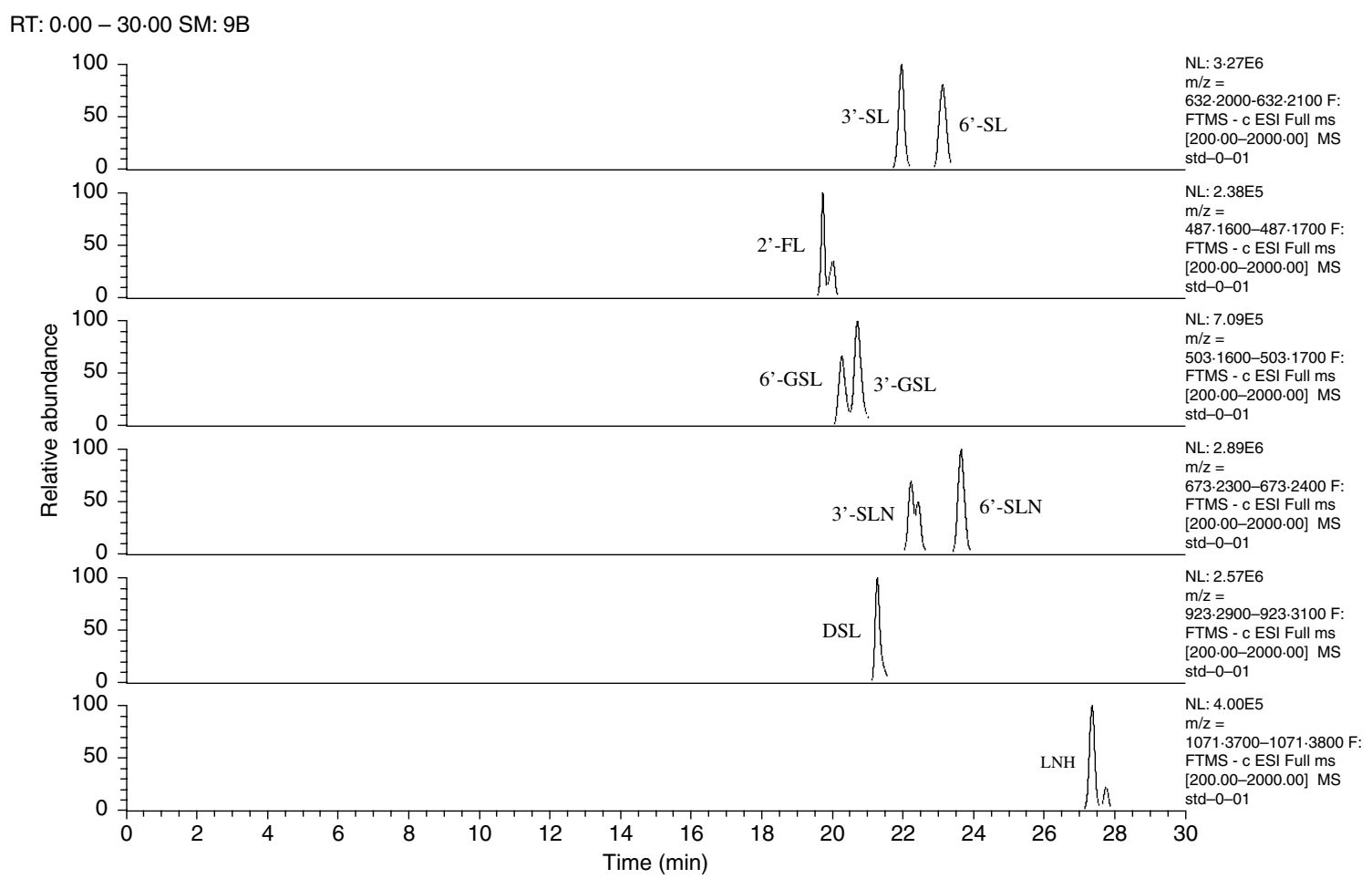

Fig. 1. Ion chromatograms of extracted oligosaccharide standards and their respective retention times (shown on the X-axis). The $m / z$ of each deprotonated ion was calculated to \pm 0.01 . SL, sialyllactose; FL, fucosyllactose; GSL, galactosyllactose; SLN, sialyl- $N$-acetyllactosamine; DSL, disialyllactose; LNH, lacto- $N$-hexaose.

S2-GIF were also effective at enhancing the growth of $L$. casei 2607 when compared with controls, lactose and GOS but not dextrose. In contrast, L. acidophillus 4461 exhibited similar growth rate when cultured in the presence of GOS, lactose, as well as S1-GIF- and S2-GIF-derived oligosaccharides. These data suggest that milk oligosaccharides derived from goats' milkbased infant formula are effective at selectively promoting the growth of health-promoting bacteria in the gut. Nonetheless, clinical trials are needed (currently being planned) to confirm these benefits in human subjects. 
(A)

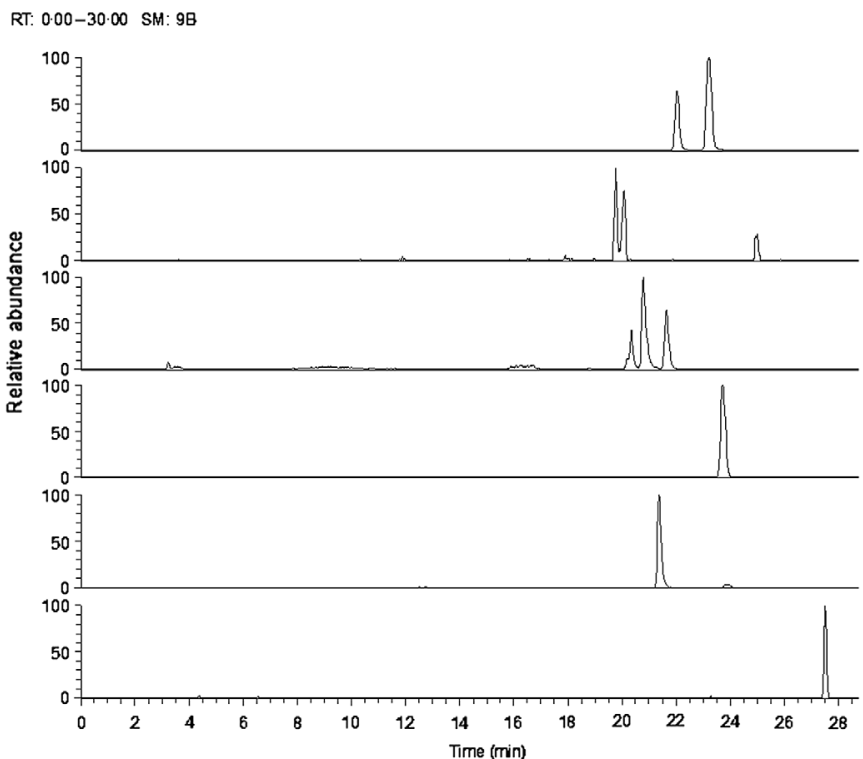

(B)

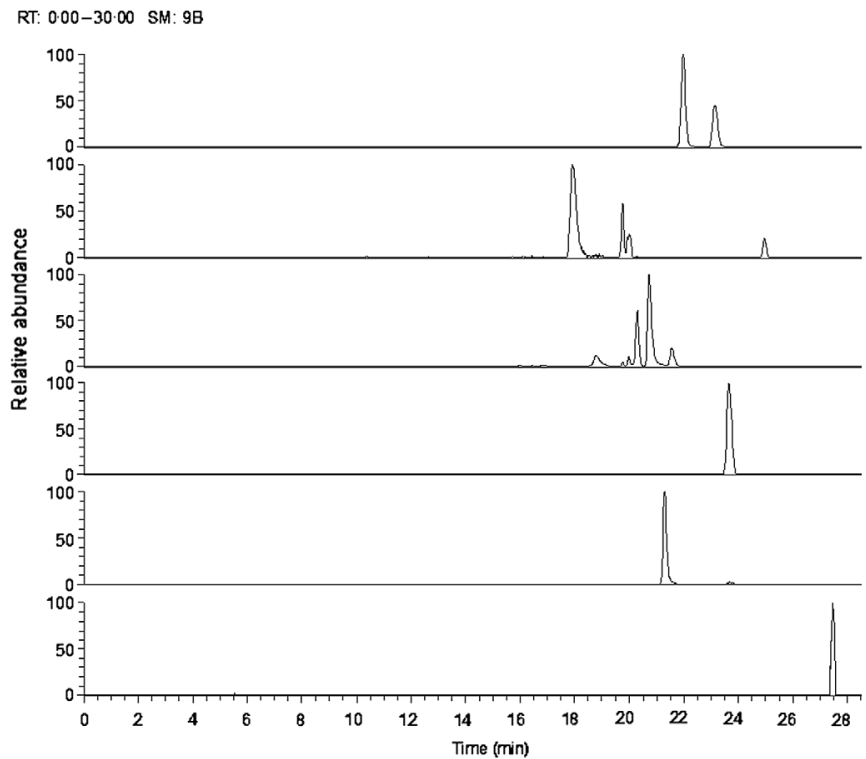

Fig. 2. Ion chromatogram of major oligosaccharides extracted from (A) goats' milk and (B) stage 1 goats' milk infant formula (S1-GIF).

(A)

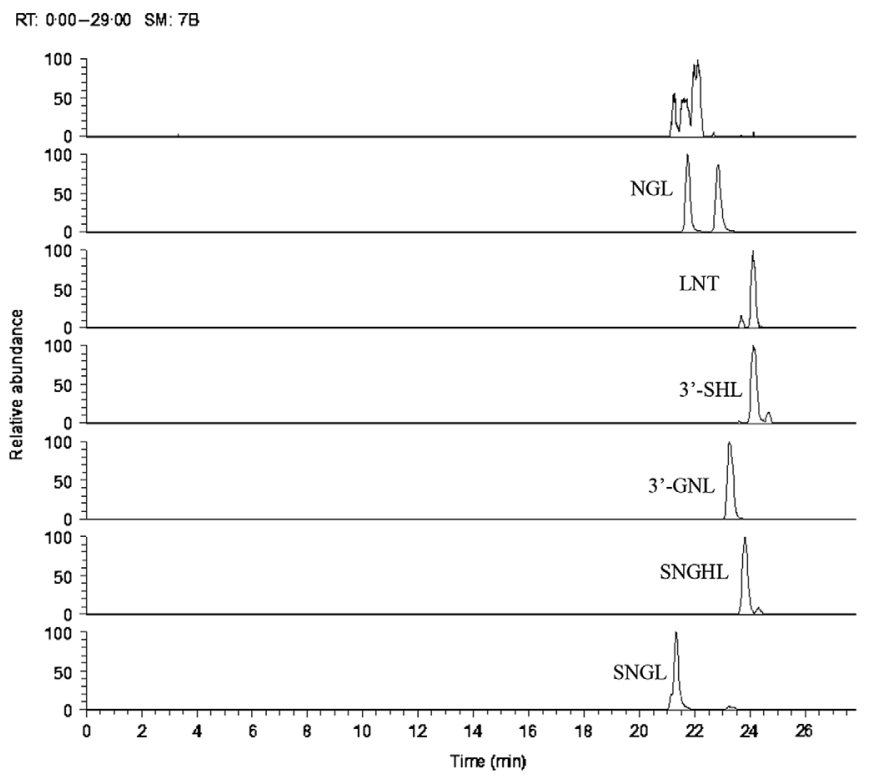

(B)

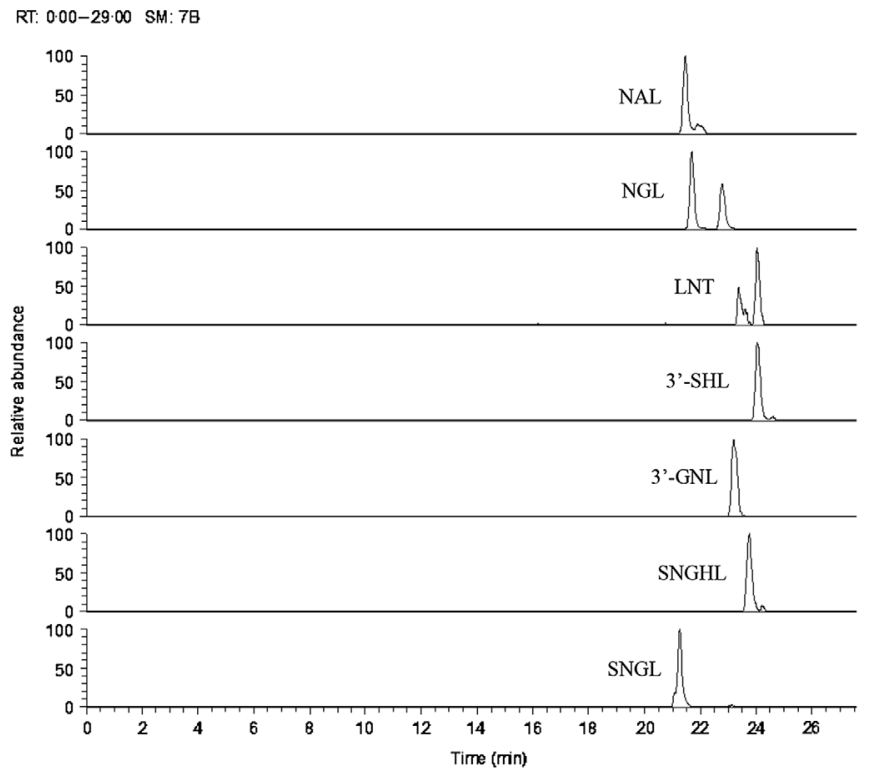

Fig. 3. Ion chromatogram of oligosaccharides (minor oligosaccharides, without standards) extracted from (A) goats' milk and (B) stage 1 goats' milk infant formula (S1-GIF). NGL, glycolyl-neuraminyl-lactose; LNT, lacto- $N$-tetraose; SHL, sialyl-galactosyl-lactose; GNL, glycolyl-neuraminyl-lactosamine; SNGHL, $N$-glycolyl-neuraminyl-hexosyl-lactose; SNGL, sialyl- $N$-glycolyl-neuraminyl-lactose; NAL, $N$-acetyl-glucosaminyl-lactose.

Goat milk oligosaccharides prevent Escherichia coli and Salmonella typhimurium adhesion to Caco-2 monolayer

Adhesion of E. coli NCTC 10418 and S. typhimurium was found to be inhibited by oligosaccharides present in S1-GIF and S2-GIF (Fig. 6) and no statistically significant differences were observed between S1 and S2 formulas. Purified GOS was also effective at preventing adhesion of pathogens to Caco-2 cells, to at least the same degree. Interestingly, oligosaccharides purified from S1-GIF, S2-GIF and GOS appeared to be slightly more efficient at inhibiting E. coli NCTC 10418 adhesion to Caco-2 cells compared with S. typhimurium, at least for the isolates we examined here. In our study, lactose had no significant protective effect.

\section{Discussion}

The diverse array of highly complex oligosaccharides present in human breast milk (HMO) is considered to be of vital importance in promoting the growth of beneficial bacteria in the infant gut, conferring protection against intestinal infections and promoting 


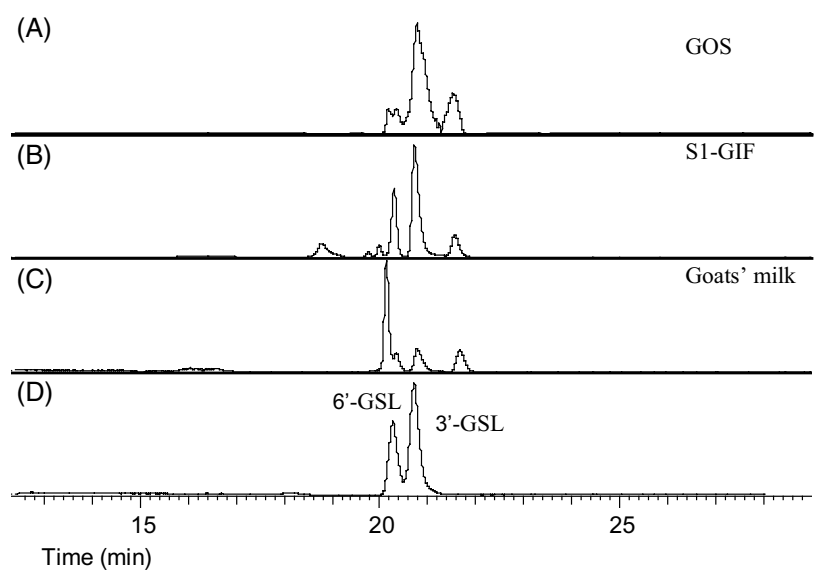

Fig. 4. Ion chromatograms of extracted oligosaccharides: (A) galactooligosaccharide (GOS), (B) stage 1 goats' milk infant formula (S1-GIF), (C) goats' milk and (D) oligosaccharide standards (3'-galactosyllactose (3'-GSL) and 6'-GSL).

(A)

Bifidobacterium animalis BB12

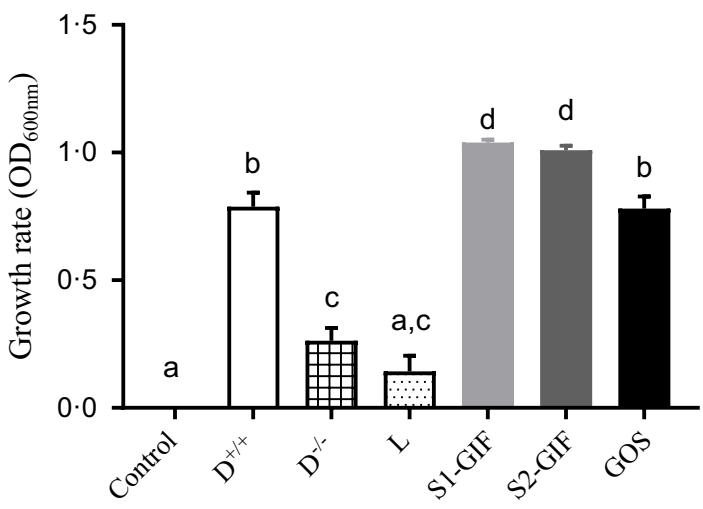

(C)

Lactobacillus casei 2607

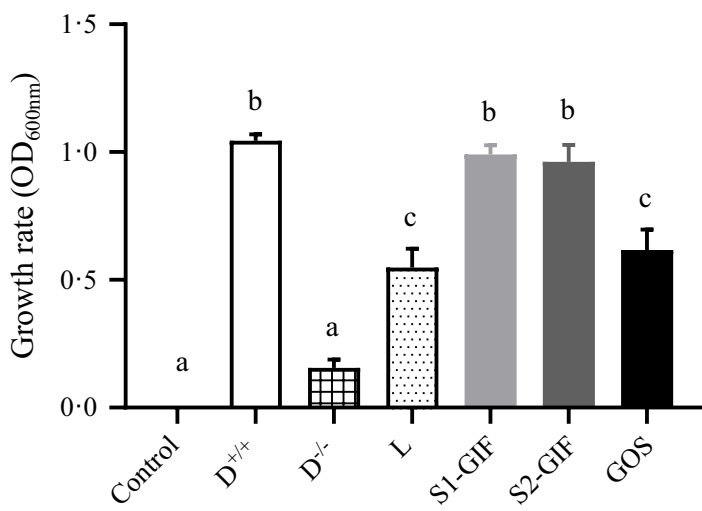

the development and maturation of the immune system ${ }^{(9,14)}$. Compared with this, oligosaccharides found in bovine and caprine milk exhibit lesser diversity and complexity, and are found in relatively smaller amounts. As a result, the amount and nature of natural milk oligosaccharides present in either caprine or bovine milk-based infant formulas, and their effectiveness in conferring gut and immune health benefits on the infant, similar to those of breast milk, remain to be determined and are of significant research interest.

The results of the present study show that goats' milk-based infant formula contains significant amounts and a diverse array of oligosaccharides, many of which are structurally similar to HMO. To our knowledge, this is the first such report showing such richness of oligosaccharides in goats' milk-based infant formula and their similarity to HMO. Fourteen oligosaccharides, including seven major oligosaccharides, were detected in goats' milk-based infant formula. Of these, five oligosaccharides are also found in human breast milk (Table 1). Goats' milk was also found to contain a similar number of oligosaccharides. Notably, the number of oligosaccharides detected in the present study is significantly lower than that reported previously in goats' milk $^{(20,36-42)}$. This is probably due to major differences in the

(B)

Bifidobacterium longum BB536

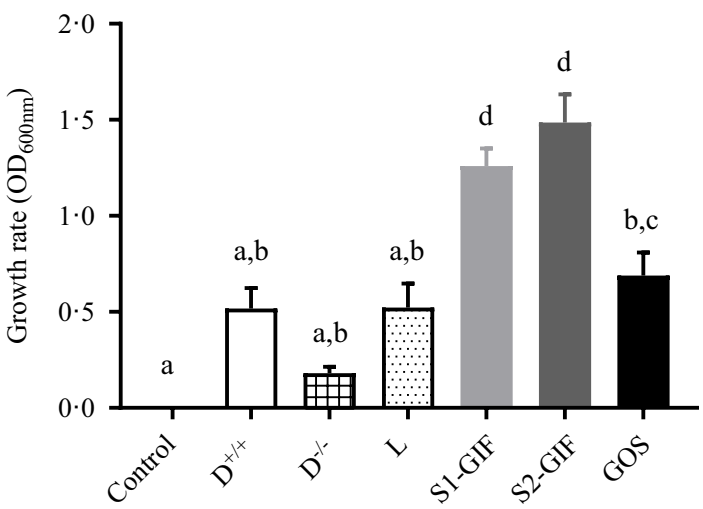

(D)

Lactobacillus acidophilus 4461

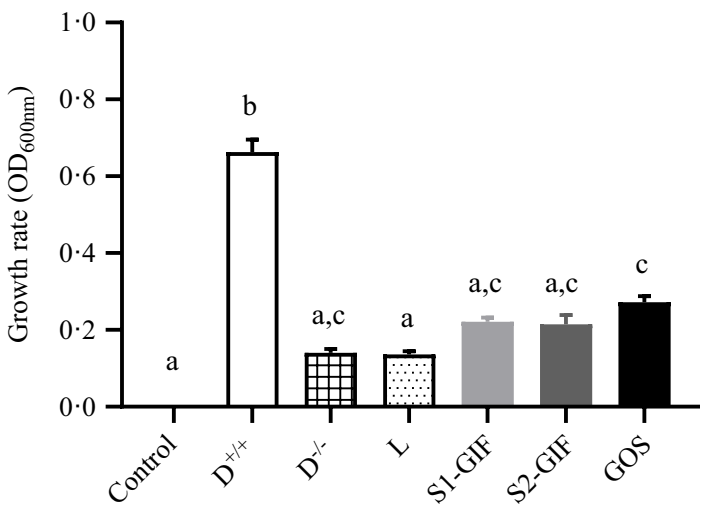

Fig. 5. Extent of growth of (A) Bifidobacterium animalis BB12, (B) B. Iongum BB536, (C) Lactobacillus casei2607, and (D) L. acidophilius 4461 after $24 \mathrm{~h}$ of co-incubation with milk oligosaccharides at $37^{\circ} \mathrm{C}$ anaerobically. D, dextrose $\left(^{+/+}\right.$indicates presence of dextrose, whilst ${ }^{-/-}$indicates absence of dextrose in bacteria culture media); L, lactose; S1-GIF, stage 1 goats' milk infant formula; S2-GIF, stage 2 goats' milk infant formula; GOS, galacto-oligosaccharide; OD, optical density. Values are means, with standard errors represented by vertical bars. a,b,c,d Mean values with unlike letters are significantly different $(P<0.05)$. 
(A)

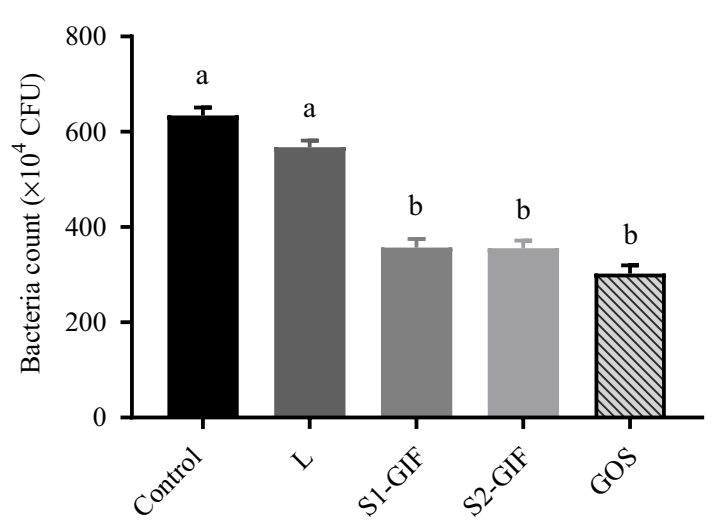

(B)

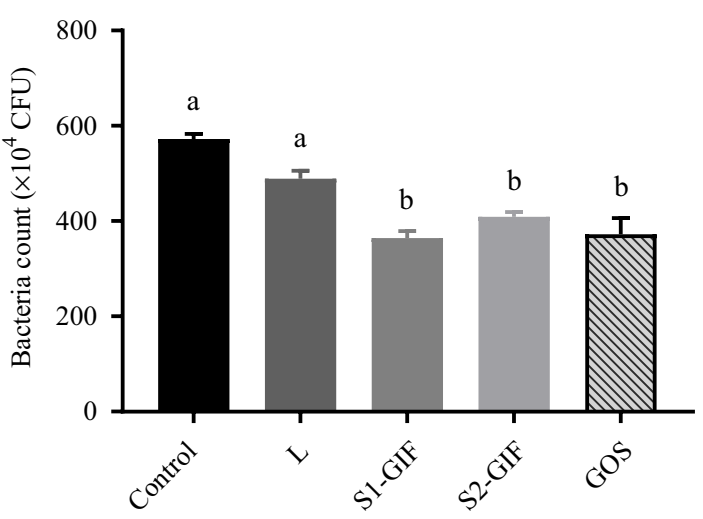

Fig. 6. Bacterial counts of (A) Escherichia coli NCTC 10418 and (B) Salmonella typhimurium adhered to Caco-2 monolayers. Pathogens were co-cultured with purified oligosaccharide for $2 \mathrm{~h}$ aerobically at $37^{\circ} \mathrm{C}$ prior to being co-incubated with Caco-2 cells for 90 min at $37^{\circ} \mathrm{C}$. L, lactose; S1-GIF, stage $1 \mathrm{goats}$ ' milk infant formula; S2-GIF, stage 2 goats' milk infant formula; GOS, galacto-oligosaccharide; CFU, colony-forming units. Values are means, with standard errors represented by vertical bars. a,b Mean values with unlike letters are significantly different $(P<0.05)$.

methods used in these studies. The present study used a high throughput method that was aimed at detecting only quantifiable oligosaccharides; the samples used in the present study involved the reconstitution of powders according to manufacturer's instructions for infant use and involved no sample concentration steps. In contrast, all earlier studies have employed methods involving significant enrichment of oligosaccharides with the aim to detect all oligosaccharides, including those present in trace amounts. Using a similar method, we detected thirty seven oligosaccharides in goats' milk in earlier studies (Leong et al., unpublished results). Similar profile and diversity of oligosaccharides detected in raw goats' milk and goats' milk-based infant formula suggests that oligosaccharides are not affected by heat treatment used during manufacture of infant formula.

As in human breast milk, fucosylated and sialylated oligosaccharides were found to be the dominant oligosaccharides present in goats' milk-based infant formula. 2'-FL is the most abundant oligosaccharide in human milk ${ }^{(36,43)}$ and is the focus of significant commercial and regulatory interest. It has been shown to play a significant role in anti-infection properties of breast milk; for example, infants with high levels of 2'-FL have been shown to exhibit greater resistance to stable toxin of E. coli and Campylobacterassociated diarrhoea ${ }^{(44)}$. Oligosaccharides resist digestion in the small intestine and reach the colon intact where they are known to influence the structure and function of gut microbiota. For example, several studies have shown that HMO are effective in selectively promoting the growth of beneficial bacteria, such as B. bifidum that dominates the gut microflora of breast fed infants ${ }^{(45-48)}$. The ability of $\mathrm{HMO}$ and a mixture of fructooligosaccharides/GOS to promote the growth of bifidobacteria and lactobacilli in vitro also has been demonstrated ${ }^{(49)}$. In the present study, goat milk oligosaccharides were found to exhibit similar prebiotic properties. They were found to be effective in promoting the growth of both Bifidobacterium and Lactobacillus species. Furthermore, oligosaccharides derived from both stage 1 and stage 2 goats' milk-based infant formula appeared to be more efficient at promoting the growth of B. longum BB536 and $L$. casei 2607 than GOS. Whether this suggests a stronger prebiotic activity of goat milk oligosaccharides compared with GOS generally or is simply a reflection of bacterial species and/ or strain selectivity in substrate utilisation is not clear ${ }^{(50)}$.

Differences in the ability of bifidobacteria and lactobacilli in their ability to utilise carbohydrates such as lactose, GOS and lactulose have also been previously reported ${ }^{(51)}$. Lactose is highly likely to be present in the GIF oligosaccharide fractions, however, the relatively insignificant growth-promoting effect of lactose, especially for bifidobacteria, compared with that for S1 and S2 fractions, suggests that the growth enhancement observed with S1 and S2 was largely due to oligosaccharides. Furthermore, the GIF was found in our study to be rich in 3'-sialyllactose, 6'-sialyllactose and 2'-FL, and the ability of B. longum and B. infantis to selectively ferment these oligosaccharides together with similarities in the gut microbiota composition of infants fed goats' milk-based infant formula compared with breast-fed babies has also been recently reported ${ }^{(52)}$.

The results of our study further showed that oligosaccharide enriched fractions prepared from both stage 1 and stage 2 goats' milk-based infant formula were effective in reducing the adherence of E. coli and S. typhimurium to Caco-2 cells, as similarly reported for $\mathrm{HMO}^{(42,47)}$. Lower incidence of gastrointestinal infections in breast-fed infants compared with formula-fed infants ${ }^{(53)}$ and a role for HMO in this protection have been reported $^{(54)}$. We found differences in the anti-adhesion efficacy against two different enteric pathogens to Caco-2 cells when preincubated and co-cultured with the same GIF oligosaccharide fractions, although further strains or isolates of these pathogens need to be tested before making a definitive conclusion. However, it is significant in this regard that Coppa et al. ${ }^{(34)}$ found species differences between E. coli, Vibrio cholerae and S. fyris in experiments using $\mathrm{HMO}$ and (like us) Caco- 2 cells. Whilst some recent studies have reported that oligosaccharides from human, cows' and goats' milk ${ }^{(55,56)}$ are effective in inhibiting the adhesion of $E$. coli to gut cells, these experiments were conducted using much higher concentrations of oligosaccharides than we used in our study, suggesting the possibility of a dose-dependent effect. In our study, GOS was at least equally as effective as goat milk 
oligosaccharides in preventing the adhesion of $E$. coli and S. typhimurium (by 52.4 and $35 \%$, respectively, compared with the negative control; see Fig. 6).

Attachment of pathogens to specific receptors on the gut epithelial cells is a critical step in host colonisation and infection. Carbohydrate chains form a major component of cell surface membranes, and lactose being a core saccharide of glycolipids represents a major binding site for bacteria ${ }^{(57,58)}$. Some pathogens also bind to lectins in attempt to colonise gut epithelial surfaces and the lectin binding requires either sialyl or fucose groups to be present on the cognate glycan binding partners ${ }^{(14)}$. The resemblance of certain GOS to saccharide-containing glycoproteins used by many pathogens to attach to intestinal cells has also been reported ${ }^{(59)}$. Due to structural similarities between goats' milk oligosaccharides and gut cell surface carbohydrate groups, these compounds, especially sialylated and fucosylated oligosaccharides, may reduce pathogen adhesion to gut epithelial cells by acting as soluble analogues of host cell receptors and/or changing the expression of such structures ${ }^{(60-62)}$. In addition, oligosaccharides have also been shown to mediate protection against intestinal pathogens by enhancing immune function $^{(11)}$. Together, these results combined with the results of our study show that oligosaccharides present in goats' milk-based infant formula have strong prebiotic and anti-infection properties and may confer protection against gastrointestinal infections to the infant. Further studies are required to confirm if this is so.

\section{Acknowledgements}

We thank Nuchev Pty Ltd for providing goat milk infant formula (Oli6) and raw goat milk samples and Dr Osaana Donker for providing Bifidobacterium and Lactobacillus strains. We also express our thanks to Justin Peace for helpful discussions.

H. G. and B. Z. had received funding from Nuchev Pty Ltd in the past to review nutritional composition and analyse oligosaccharides in goats' milk. The research costs were covered by RMIT University funding for PhD student (A. L.) and an Entrepreneurs' Programme - Innovation Connections Grant between the Australian Government and Nuchev Pty Ltd (ICG number 55140). Nuchev Pty Ltd had no role in the design of the study; in the collection, analyses or interpretation of data; in the writing of the manuscript; and in the decision to publish the results.

Contributions of authors were as follows: conceptualisation: H. G., B. Z. and A. L.; data curation: A. L.; formal analysis: A. L. and Z. L.; investigation: A. L.; methodology: A. L., Z. L., C. P., H. G., B. Z. and H. A.; resources: H. G.; supervision: H. G. and B. Z.; validation: A. L.; writing original draft: A. L.; Writing-review and editing: H. G., C. P. and B. Z.

The authors received co-funding from Nuchev Pty Ltd for this research.

\section{References}

1. World Health Organization (WHO) (2011) Exclusive Breastfeeding for Six Months Best for Babies Everywhere. http://www.who.int/mediacentre/news/statements/2011/ breastfeeding_20110115/en/ (accessed March 2017).

2. Urashima T \& Taufik E (2011) Oligosaccharides in milk: their benefits and future utilization. Media Peternakan 33, 189-197.
3. Sanz Ceballos L, Morales ER, de la Torre Adarve G, et al. (2009) Composition of goat and cow milk produced under similar conditions and analyzed by identical methodology. J Food Comp Anal 22, 322-329.

4. Le Parc A, Dallas DC, Duaut S, et al. (2014) Characterization of goat milk lactoferrin N-glycans and comparison with the $\mathrm{N}$-glycomes of human and bovine milk. Electrophoresis $\mathbf{3 5}$, 1560-1570.

5. Gabrielli O, Zampini L, Galeazzi T, et al. (2011) Preterm milk oligosaccharides during the first month of lactation. Pediatrics 128, e1520-e1531.

6. Ruhaak LR \& Lebrilla CB (2012) Advances in analysis of human milk oligosaccharides. Adv Nutr 3, 406s-414s.

7. Asakuma S, Hatakeyama E, Urashima T, et al. (2011) Physiology of consumption of human milk oligosaccharides by infant gut-associated bifidobacteria. J Biol Chem 286, 34583-34592.

8. Bode L (2006) Recent advances on structure, metabolism, and function of human milk oligosaccharides. J Nutr 136, 2127-2130.

9. Bode L (2012) Human milk oligosaccharides: every baby needs a sugar mama. Glycobiology 22, 1147-1162.

10. Gopal PK \& Gill H (2000) Oligosaccharides and glycoconjugates in bovine milk and colostrum. Br J Nutr 84, Suppl. 1, S69-S74.

11. Triantis V, Bode L \& Van Neerven R (2018) Immunological effects of human milk oligosaccharides. Front Pediatr 6, 190.

12. Sela DA \& Mills DA (2010) Nursing our microbiota: molecular linkages between bifidobacteria and milk oligosaccharides. Trends Microbiol 18, 298-307.

13. Bode L \& Jantscher-Krenn E (2012) Structure-function relationships of human milk oligosaccharides. Adv Nutr 3, 383S-391S.

14. Newburg DS, Ruiz-Palacios GM \& Morrow AL (2005) Human milk glycans protect infants against enteric pathogens. Annu Rev Nutr 25, 37-58.

15. Institute of Medicine (2004) Infant Formula: Evaluating the Safety of New Ingredients, pp. 41-54, Washington, DC: The National Academies Press.

16. Jandal JM (1996) Comparative aspects of goat and sheep milk. Small Rumin Res 22, 177-185.

17. Park YW, Juárez M, Ramos M, et al. (2007) Physico-chemical characteristics of goat and sheep milk. Small Rumin Res $\mathbf{6 8}$, 88-113.

18. Kiskini A \& Difilippo E (2013) Oligosaccharides in goat milk: structure, health effects and isolation. Cell Mol Biol (Noisy-legrand) 59, 25-30.

19. Raynal-Ljutovac K, Lagriffoul G, Paccard P, et al. (2008) Composition of goat and sheep milk products: an update. Small Rumin Res 79, 57-72.

20. Martinez-Ferez A, Rudloff S, Guadix A, et al. (2006) Goats' milk as a natural source of lactose-derived oligosaccharides: isolation by membrane technology. Int Dairy J 16, 173-181.

21. Moro GE, Stahl B, Fanaro S, et al. (2005) Dietary prebiotic oligosaccharides are detectable in the faeces of formula-fed infants. Acta Paediatr 94, 27-30.

22. Oozeer R, van Limpt K, Ludwig T, et al. (2013) Intestinal microbiology inearly life: specific prebiotics canhave similarfunctionalities as human-milk oligosaccharides. Am J Clin Nutr 98, 561s-571s.

23. Holma R, Juvonen P, Asmawi MZ, et al. (2002) Galactooligosaccharides stimulate the growth of bifidobacteria but fail to attenuate inflammation in experimental colitis in rats. Scand J Gastroenterol 37, 1042-1047.

24. Moreau NM, Martin LJ, Toquet CS, et al. (2003) Restoration of the integrity of rat caeco-colonic mucosa by resistant starch, but not by fructo-oligosaccharides, in dextran sulfate sodiuminduced experimental colitis. Br J Nutr 90, 75-85.

25. Böhm A, Kaiser I, Trebstein A, et al. (2005) Heat-induced degradation of inulin. Eur Food Res Technol 220, 466-471. 
26. Matusek A, Merész P, Khanh Diem Le T, et al. (2009) Effect of temperature and $\mathrm{pH}$ on the degradation of fructooligosaccharides. Eur Food Res Technol 220, 355-365.

27. Skanderby M, Westergaard V, Patridge A, et al. (2009) Dried milk products. In Dairy Powders and Concentrated Products, 1st ed., pp. 180-234 [A Tamime, editor]. Oxford: Blackwell Publishing Ltd.

28. Liu Z, Moate P, Cocks B, et al. (2014) Simple liquid chromatography-mass spectrometry method for quantification of major free oligosaccharides in bovine milk. Eur Food Res Technol 228, 355-365.

29. Otieno DO \& Shah NP (2007) Endogenous $\beta$-glucosidase and $\beta$-galactosidase activities from selected probiotic micro-organisms and their role in isoflavone biotransformation in soymilk. $J$ Appl Microbiol 103, 910-917.

30. Pham TT \& Shah NP (2008) Skim milk powder supplementation affects lactose utilization, microbial survival and biotransformation of isoflavone glycosides to isoflavone aglycones in soymilk by Lactobacillus. Food Microbiol 25, 653-661.

31. Pham TT \& Shah NP (2008) Effect of lactulose on biotransformation of isoflavone glycosides to aglycones in soymilk by lactobacilli. J Food Sci 73, M158-M165.

32. De Man J, Rogosa D \& Sharpe ME (1960) A medium for the cultivation of lactobacilli. J Appl Bacteriol 23, 130-135.

33. Altamimi M, Abdelhay O \& Rastall RA (2016) Effect of oligosaccharides on the adhesion of gut bacteria to human HT-29 cells. Anaerobe 39, 136-142.

34. Coppa GV, Zampini L, Galeazzi T, et al. (2006) Human milk oligosaccharides inhibit the adhesion to Caco-2 cells of diarrheal pathogens: Escherichia coli, Vibrio cholerae, and Salmonella fyris. Pediatr Res 59, 377-382.

35. Wu S, Tao N, German JB, et al. (2010) Development of an annotated library of neutral human milk oligosaccharides. J Proteome Res 9, 4138-4151.

36. Albrecht S, Lane JA, Marino K, et al. (2014) A comparative study of free oligosaccharides in the milk of domestic animals. $\mathrm{BrJ}$ Nutr 111, 1313-1328.

37. Chaturvedi P \& Sharma CB (1988) Goat milk oligosaccharides: purification and characterization by HPLC and high-field ${ }^{1}$ H-NMR spectroscopy. Biochim Biophys Acta 967, 115-121.

38. Martín-Ortiz A, Salcedo J, Barile D, et al. (2016) Characterization of goat colostrum oligosaccharides by nanoliquid chromatography on chip quadrupole time-of-flight mass spectrometry and hydrophilic interaction liquid chromatography-quadrupole mass spectrometry. J Chromat A 1428, 143-153.

39. Mehra R \& Kelly P (2006) Milk oligosaccharides: structural and technological aspects. Int Dairy J 16, 1334-1340.

40. Meyrand M, Dallas DC, Caillat H, et al. (2013) Comparison of milk oligosaccharides between goats with and without the genetic ability to synthesize $\alpha$ s1-casein. Small Rumin Res 113, 411-420.

41. Urashima T, Bubb WA, Messer M, et al. (1994) Studies of the neutral trisaccharides of goat (Capra bircus) colostrum and of the one-and two-dimensional ${ }^{1} \mathrm{H}$ and ${ }^{13} \mathrm{C}$ NMR spectra of 6'-N-acetylglucosaminyllactose. Carbohyd Res 262, 173-184.

42. Tangvoranuntakul P, Gagneux P, Diaz S, et al. (2003) Human uptake and incorporation of an immunogenic nonhuman dietary sialic acid. Proc Natl Acad Sci U S A 100, 12045-12050.

43. Thurl S, Munzert M, Boehm G, et al. (2017) Systematic review of the concentrations of oligosaccharides in human milk. Nutr Rev 75, 920-933.

44. Newburg DS, Ruiz-Palacios GM, Altaye M, et al. (2004) Innate protection conferred by fucosylated oligosaccharides of human milk against diarrhea in breastfed infants. Glycobiology $\mathbf{1 4}$ 253-263.

45. LoCascio RG, Desai P, Sela DA, et al. (2010) Broad conservation of milk utilization genes in Bifidobacterium longum subsp. infantis as revealed by comparative genomic hybridization. Appl Environ Microbiol 76, 7373-7381.

46. Strum JS, Kim J, Wu S, et al. (2012) Rapid identification and accurate quantitation of biological oligosaccharide mixtures. Anal Chem 84, 7793-7801.

47. Thongaram T, Hoeflinger JL, Chow J, et al. (2017) Human milk oligosaccharide consumption by probiotic and human-associated bifidobacteria and lactobacilli. J Dairy Sci 100, 7825-7833.

48. Turroni F, Bottacini F, Foroni E, et al. (2010) Genome analysis of Bifidobacterium bifidum PRL2010 reveals metabolic pathways for host-derived glycan foraging. Proc Natl Acad Sci U S A 107, 19514-19519.

49. Shen Q, Tuohy KM, Gibson GR, et al. (2011) In vitro measurement of the impact of human milk oligosaccharides on the faecal microbiota of weaned formula-fed infants compared to a mixture of prebiotic fructooligosaccharides and galactooligosaccharides. Lett Appl Microbiol 52, 337-343.

50. Garrido D, Ruiz-Moyano S, Jimenez-Espinoza R, et al. (2013) Utilization of galactooligosaccharides by Bifidobacterium longum subsp. infantis isolates. Food Microbiol 33, 262-270.

51. Watson D, O'Connell Motherway M, Schoterman MH, et al. (2013) Selective carbohydrate utilization by lactobacilli and bifidobacteria. J Appl Microbiol 114, 1132-1146.

52. Tannock GW, Lawley B, Munro K, et al. (2013) Comparison of the compositions of the stool microbiotas of infants fed goat milk formula, cow milk-based formula, or breast milk. Appl Environ Microbiol 79, 3040-3048.

53. Victora CG, Bahl R, Barros AJ, et al. (2016) Breastfeeding in the 21st century: epidemiology, mechanisms, and lifelong effect. Lancet 387, 475-490.

54. Morrow AL, Ruiz-Palacios GM, Jiang X, et al. (2005) Humanmilk glycans that inhibit pathogen binding protect breast-feeding infants against infectious diarrhea. J Nutr 135, 1304-1307.

55. Facinelli B, Marini E, Magi G, et al. (2018) Breast milk oligosaccharides: effects of $2^{\prime}$-fucosyllactose and $6^{\prime}$-sialyllactose on the adhesion of Escherichia coli and Salmonella fyris to Caco-2 cells. J Matern Fetal Neonatal Med 32, 2950-2952.

56. Urakami H, Saeki M, Watanabe Y, et al. (2018) Isolation and assessment of acidic and neutral oligosaccharides from goat milk and bovine colostrum for use as ingredients of infant formulae. Int Dairy J 83, 1-9.

57. Karlsson KA (1989) Animal glycosphingolipids as membrane attachment sites for bacteria. Ann Rev Biochem 58, 309-350.

58. Sharon N \& Ofek I (2000) Safe as mother's milk: carbohydrates as future anti-adhesion drugs for bacterial diseases. GlycoconjJ 17, 659-664.

59. Kunz C, Rudloff S, Baier W, et al. (2000) Oligosaccharides in human milk: structural, functional, and metabolic aspects. Ann Rev Nutr 20, 699-722.

60. Ebersbach T, Andersen JB, Bergstrom A, et al. (2012) Xylo-oligosaccharides inhibit pathogen adhesion to enterocytes in vitro. Res Microbiol 163, 22-27.

61. Newburg DS (1997) Do the binding properties of oligosaccharides in milk protect human infants from gastrointestinal bacteria? J Nutr 127, 980s-984s.

62. Ofek I, Hasty DL \& Sharon N (2003) Anti-adhesion therapy of bacterial diseases: prospects and problems. FEMS Immunol Med Microbiol 38, 181-191. 